196 words in the abstract 4060 words in the text

3 figures and 3 tables

1 supplemental material

\title{
The effects of cognitive-behavioral therapy on intrinsic functional brain networks in adults with attention-deficit/hyperactivity disorder
}

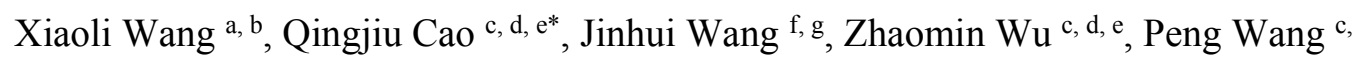

d, e, Li Sun ${ }^{c, d, e}$, Taisheng Cai ${ }^{a^{*}}$, Yufeng Wang ${ }^{\text {c, d, e }}$

${ }^{\text {a }}$ Medical Psychological Institute of The Second Xiangya Hospital, Central South

University, Changsha, Hunan Province 410011, China

${ }^{\mathrm{b}}$ Guangdong Provincial Corps Hospital of Chinese People's Armed Police Forces,

Guangzhou Medical University, Guangzhou City, Guangdong Province 510505,

China

${ }^{\mathrm{c}}$ Institute of Mental Health, Peking University, Beijing 100191, China

d Peking University Sixth Hospital, Beijing 100191, China

${ }^{\mathrm{e}}$ Key Laboratory of Mental Health, Ministry of Health (Peking University), Beijing

100191, China

${ }^{\mathrm{f}}$ Center for Cognition and Brain Disorders, Hangzhou Normal University, Hangzhou, 311121, China

${ }^{\mathrm{g}}$ Zhejiang Key Laboratory for Research in Assessment of Cognitive Impairments, Hangzhou, 311121, China

Corresponding authors: Qingjiu Cao, Institute of Mental Health, Peking University, Huayuanbeilu 51, Haidian District, Beijing 100191, China. Email: caoqingjiu@bjmu.edu.cn or Taisheng Cai, Medical Psychological Institute of The 
Second Xiangya Hospital, Central South University, Middle Ren-Min Road 139,

Changsha, Hunan 410011, China. Email: caitigers@sina.com. 


\begin{abstract}
Cognitive-behavioral therapy (CBT) is an efficacious psychological treatment for adults with attention-deficit/hyperactivity disorder (ADHD), but the neural processes underlying the benefits of CBT are not well understood. This study aims to unravel psychosocial mechanisms for treatment ADHD by exploring the effects of CBT on functional brain networks. Ten adults with ADHD were enrolled and resting-state functional magnetic resonance imaging scans were acquired before and after a 12-session CBT. Twelve age- and gender-matched healthy controls were also scanned. We constructed whole-brain functional connectivity networks using graph-theory approaches and further computed the changes of regional functional connectivity strength (rFCS) between pre- and post-CBT in ADHD for measuring the effects of CBT. The results showed that rFCS was increased in the fronto-parietal network and cerebellum, the brain regions that were most often affected by medication, in adults with ADHD following CBT. Furthermore, the enhanced functional coupling between bilateral superior parietal gyrus was positively correlated with the improvement of ADHD symptoms following CBT. Together, these findings provide evidence that CBT can selectively modulate the intrinsic network connectivity in the fronto-parietal network and cerebellum and suggest that the CBT may share common brain mechanism with the pharmacology in adults with ADHD.
\end{abstract}

Key words: ADHD, CBT, resting-state fMRI, graph theory, fronto-parietal network

\footnotetext{
Abbreviations: ADHD, attention-deficit/hyperactivity disorder; CBT, cognitive-behavioral therapy; HC, healthy control; r-fMRI, resting-state functional magnetic resonance imaging; rFCS, regional functional connectivity strength; SPG, superior parietal gyrus
} 


\section{Introduction}

Attention -deficit/hyperactivity disorder (ADHD), which is characterized primarily by behavioral symptoms of inattention, hyperactivity, and impulsivity, is a common disorder of childhood onset that may persist into adulthood. This disorder is now increasingly recognized as developmental impairments of cognitive functions, including inhibition, working memory, self-mobilization, organization, and time management (Brown, 2008; Stavro, Ettenhofer, \& Nigg, 2007). Longitudinal follow-up studies have extensively indicated persisting impairments in academic, occupational, social, and emotional domains of functioning in adults with ADHD (Biederman, et al., 2010). In addition, adults with ADHD are at very high risk for comorbid disorders including anxiety, depression, and personality disorders (Miller, Nigg, \& Faraone, 2007).

Although ADHD is considered as a neurobiological disorder, the neural basis of this disorder remains unknown. Accumulating evidence suggests that the abnormalities of prefrontal-striatal-cerebellar circuit, which subserves the cognitive functions, should be considered as an important neuropsychological correlate of ADHD (Krain \& Castellanos, 2006). However, recent studies from resting-state functional magnetic resonance imaging (r-fMRI) have revealed several other large-scale neural networks, such as the fronto-parietal network, the dorsal and ventral attentional networks and the default network, whose abnormalities are also involved in the pathophysiology of ADHD (Castellanos \& Proal, 2012) and related to the severity of ADHD symptoms (Choi, Jeong, Lee, \& Go, 2013; Cocchi, et al., 2012; Tomasi \& Volkow, 2012).

Pharmacological interventions are the first-line choices for treatment of adults with ADHD; however, many adults with ADHD cannot or will not take medications while others show a poor response (Lofthouse, Arnold, Hersch, Hurt, \& DeBeus, 2012). Psychosocial interventions, such as cognitive-behavioral therapy (CBT), are also established treatments for ADHD. These approaches help those who cannot tolerate, fail to respond to, or refuse to try medications. 
Previous randomized controlled trials (RCTs) have demonstrated that CBT can augment the outcome of medication for adults with ADHD (Emilsson, et al., 2011; Solanto, et al., 2010; S. A. Safren, et al., 2005; S. A. Safren, et al., 2010; Stevenson, Whitmont, Bornholt, Livesey, \& Stevenson, 2002; Young, et al., 2015) and have comparable effects with medication for symptom reduction in adults with ADHD (Weiss, et al., 2012). Given that CBT for adult ADHD emphasizes the use of coping strategies with which to manage the effects of ADHD, the cognitive functions of patients receiving this treatment are thought to be improved (Ramsay, 2007), and a previous study had proved this inference (Stevenson, et al., 2002). Nonetheless, the neural correlations of CBT to reduce the ADHD symptoms in adults with ADHD remain unknown.

Studying brain mechanisms of CBT in ADHD could significantly enhance knowledge about neurophysiology of ADHD and improve the specificity interventions. In this study, using the r-fMRI, we explored the CBT effects on whole-brain connectivity as an important step in unraveling psychosocial mechanisms for treatment ADHD at the level of neural circuits. We constructed whole-brain functional connectivity networks by measuring temporal correlations of every pair of brain voxels and further analyzed their changes in ADHD following CBT. Specifically, we investigated the changes of regional functional connectivity strength $(\mathrm{rFCS})$, a centrality measure from graph theory, which captures functional integration strength between a given voxel and that of the rest of the brain. Finally, we examined the relationship between the changes of rFCS and functional connectivity and the alterations of clinical and cognitive measures in ADHD group.

\section{Methods and Materials}

\section{Participants, clinical assessment and procedure}

The participants included 10 adults with ADHD (age 18-31 years) who participated a 12- session CBT trial and took MRI scans within one week before and after CBT, and 12 healthy control (HC) subjects (age 19-32 years) who took once MRI scan. Nine 
patients were medication naïve; one patient had a history of stimulant treatment and withdrawal of the medication half a year before the study. Eight patients met the criteria for the predominantly inattentive subtype, and the other 2 met the criteria for the combined (inattentive/hyperactive-impulsive) subtype (demographic and clinical characteristics are summarized in Table 1). All participants met the following criteria: (1) right-handedness, (2) have no lifetime history of head trauma with loss of consciousness, (3) no history of neurological illness or other serious psychical disease, (4) the full scores of Wechsler Intelligence Scale for Chinese Adult-Revised (WISCC-R) higher than 80 . The patients were selected from a pool of 36 potential participants recruited through online advertising or from outpatient clinics in Peking University Sixth Hospital. At least two clinicians interviewed the patients to ensure that they met Diagnostic and Statistical Manual of Mental Disorders (DSM-IV; APA, 2000) criteria for adult ADHD and confirmed the diagnosis using the Conner's Adult ADHD Diagnostic Interview for DSM-IV (CAARID; Conners, Erhardt, \& Sparrow, 1999). All patients presented at least six of the nine inattention symptoms with or without six of nine hyperactivity/impulsive symptoms at the moment of interviewed and during childhood (before age 7 years) assessed by CAARID. The HC were also assessed by CAARID to insure they had no lifetime or current ADHD diagnosis. The Structural Clinical Interview for DSM-IV Axis I Disorders (SCID-I, First, Spitzer, Gibbon, \& Williams, 2012) was used to ascertain that all the participants have no Axis I cormorbidity. This study was approved by the Research Ethics Review Board of the Peking University Sixth Hospital, and by the Institutional Review Board of the State Key Laboratory of Cognitive Neuroscience and Learning, Beijing Normal University.

The ADHD Rating Scale-IV (ADHD RS-IV), a 4-point severity grid (score of 1 indicates ADHD not present and score of 4 indicated severe ADHD) which contains all the inattention and hyperactivity/impulsivity symptoms of ADHD according to DSM-IV, were self-reported by the patients pre- and post- CBT and HC to assess the severity of the ADHD symptoms (DuPaul, Power, Anastopoulos, \& Reid, 1998). The 
Mandarin version of the Behavior Rating Inventory of Executive Function, Adult Version (BRIEF-A), which consists of 86 items based on theoretical and basic definitions of executive function construct (Gioia, Isquith, Guy, \& Gerard, 2000), was used to assess the everyday executive function in adult with ADHD pre- and post-CBT and HC. The patients were asked to rate the items related to their everyday behavior on a 3 -point Likert scale $(1=$ never, $2=$ sometimes, $3=$ often $)$. BRIEF-A includes eight subscales: initiate, working memory, plan/organize, organization of material, monitor, inhibit, shift, and emotional control. A higher score indicates more problems in that area.

The open-label CBT was administered in 12 once-weekly sessions lasting approximately 70 minutes each. The CBT approaches were based on Safren's

protocol (S. A. Safren, 2006; Steven A Safren, Perlman, Sprich, \& Otto, 2005). Three core modules and 1 optional module were included in this program. The first module (4 sessions) focused on psycho-education about ADHD and training in organization and planning. The second module ( 2 sessions) involved learning skills to reduce distractibility. The third module ( 3 sessions) was cognitive restructuring. The optional modules included one session of application of skills to procrastination, one session of relapse prevention and one session including the patient's family member for support. Therapists were clinical psychologist and postdoctoral-level clinical psychology fellows.

\section{Data Acquisition}

All imaging data were acquired on a Siemens Trio 3-Tesla scanner (Siemens, Erlangen, Germany) at the State Key Laboratory of Cognitive Neuroscience and Learning, Beijing Normal University. The r-fMRI images were obtained using an echo-planer imaging (EPI) sequence with the following parameters: 33 axial slices, repeating time $(\mathrm{TR})=2000 \mathrm{~ms}$, echo time $(\mathrm{TE})=30 \mathrm{~ms}$, flip angle $=90^{\circ}$, slice thickness $/$ skip $=3.5 / 0.7 \mathrm{~mm}$, field of view $(\mathrm{FOV})=200 \times 200 \mathrm{~mm}^{2}$, matrix $=64 \times 64$, 240 volumes. High-resolution T1-weighted structural images were then acquired 
using a spoiled gradient-recalled sequence covering the whole brain with the following parameters: 128 sagittal slices, $\mathrm{TR}=2530 \mathrm{~ms}$, $\mathrm{TE}=3.39 \mathrm{~ms}$, flip angle $=$ $7^{\circ}$, slice thickness $/ \mathrm{skip}=1.33 / 0 \mathrm{~mm}, \mathrm{FOV}=256 \times 256 \mathrm{~mm} 2$, matrix $=256 \times 192$.

\section{Data Analysis}

Imaging preprocessing

Imaging preprocessing was conducted using SPM8 (SPM, www.fil.ion.ucl.ac.uk/spm) and Data Processing Assistant for R-fMRI (Chao-Gan \& Yu-Feng, 2010) toolkits. Briefly, the first 10 volumes for each individual were discarded to allow the signal to reach equilibrium and the participants to adapt to scanning noise. The remaining volumes were corrected for the acquisition time delay between slices and then realignment was performed to correct the head motion. Participants with head motion $>3 \mathrm{~mm}$ of translation or $>3$ degree of rotation in any direction were excluded from further analysis. Subjects demonstrated no significant group differences in head-motion parameters at each direction between groups (pre-CBT ADHD vs HC and post-CBT ADHD vs HC) and conditions (pre-CBT ADHD vs post-CBT ADHD). Then the functional data were normalized to the standard EPI template in Montreal Neurological Institute (MNI) space by using 12-parameter affine transformation and nonlinear deformation, and resampled to $3 \mathrm{~mm}$ isotropic voxels. The normalized images were spatially smoothed with a $4 \mathrm{~mm}$ full width at half maximum Gaussian kernel. The datasets were then underwent removal of linear trend and temporal band-pass filtering in a frequency range of 0.01 to $0.08 \mathrm{~Hz}$. Subsequently, several nuisance signals including head motion, global signal, and signal from the cerebrospinal fluid and white matter were regressed from the data.

\section{rFCS calculation}

After these preprocessing procedures, whole-brain functional connectivity analyses were performed in a similar manner to our previous study (X. Wang, et al., 2014) to derive individual rFCS maps. First, Pearson's correlation coefficients were computed between the time series of all pairs of gray matter (GM) voxels within a GM mask 
obtained by thresholding the GM probability map in SPM8 (cut-off value $=0.2$ ). Thus, a whole-brain functional connectivity matrix was generated for each participant. Then rFCS of a given voxel was computed as the sum of positive connections $(r \geq 0.3)$ between the voxel and all other voxels in the GM mask. We excluded negative correlations considering the ambiguous interpretation of negative correlations after removal of global signal (Murphy, Birn, Handwerker, Jones, \& Bandettini, 2009). A relatively higher threshold was chosen to eliminate voxels with weak correlations attributable to signal noise. We also analyzed $\mathrm{rFCS}$ with different thresholds of $\mathrm{r}=0.2$ and 0.4 , respectively, to evaluate the effects of different correlation thresholds on the results.

\section{Seed-based functional connectivity}

To examine the detailed rFCS alternations about treatment effects in ADHD group, we performed seed-based functional connectivity analysis in pre- and post-CBT ADHD group. We selected region of interest (ROI) by overlapping two between-group comparison results (pre-CBT ADHD vs post-CBT ADHD and pre-CBT ADHD vs HC). The ROIs selected by this method represent the brain regions that not only underline the pathophysiology but also have association with the neural effects of CBT in ADHD. We only compared the positive FC between two conditions in ADHD.

\section{Statistical analyses}

Between-group differences in age and full-scale IQ were compared by two-sample t-tests in SPSS 13.0 (http://www.spss.com). The changes of severity of ADHD symptoms and scores of BRIEF-A (and subscales) in ADHD pre- and post-CBT were calculated by paired $t$-test. Sex composition between ADHD and controls was compared using a Pearson Chi-square test (two-tailed). Multivariate analysis of variance (MANOVA) was performed to assess the differences of the scores of eight BRIEF-A subscales between groups (pre-CBT ADHD vs HC and post-CBT ADHD vs HC), using age, gender, IQ, full ADHD RS-IV score as covariates. A significance 
threshold of $P=0.05 / 2(0.025)$ and $P=0.05 / 8(0.006)$ was used to account for multiple comparison issues of ADHD RS-IV and BRIEF-A subscales, respectively. For $\mathrm{rFCS}$, voxel-wise two-sample t-test was employed to determine differences between the ADHD group (pre- and post-CBT, respectivity) and $\mathrm{HC}$ with age, gender,

IQ as covariates using Resting-State fMRI Data Analysis Toolkit (Song, et al., 2011). Then a voxel-wise paired $t$-test was performed to examine the CBT effects between pre- and post-CBT in the ADHD group. Voxels with $P<0.05$ and cluster size $>123$ voxels $\left(3321 \mathrm{~mm}^{3}\right)$, which resulted in a corrected threshold of $P<0.005$ determined by AlphaSim in AFNI (rmm=5; http://afni.nih.gov/afni/docpdf/AlphaSim.pdf), were regarded as significant differences between two groups or two conditions for rFCS and FC analysis.

Then, we performed correlation analyses between the mean changes (pre-CBT minus post-CBT) in ADHD symptoms and BRIEF-A scores (including 6 variables, i.e., scores of total ADHD RS-IV and inattention and hyperactivity/impulsivity subscale, as well as BRIEF-A and its organization of material and monitor factors which showed significant differences between pre- and post-CBT in ADHD) and that of brain measurements (i.e., rFCS and seed-based FC changes between pre- and post-CBT) in the brain areas showing significant treatment effects in ADHD group. The significant level was set at $P<0.05 / 6(0.008)$ to account for a multiple comparison issue.

\section{Methodological considerations}

In this study, we used a single correlation coefficient threshold of 0.3 to eliminate weak correlations possibly arising from noise signal during the rFCS analysis. To examine whether the reported results depended on the choices of different correlation thresholds, we recomputed $\mathrm{rFCS}$ using two other correlation thresholds (i.e., 0.2 and 0.4 ) and performed statistical analysis.

\section{Results}




\section{Demographic, clinical and neuropsychological data}

There were no significant differences in gender distribution $(P=0.83)$, age $(P=0.49)$, or IQ $(P=0.83)$ between the adults with ADHD and HC. As expected, however, compared with the HC, the ADHD group got higher scores in ADHD RS-IV $(P<$ $0.001)$ and BRIEF-A $(P<0.001)$ and in all its subscales $(P \leq 0.005)$ except for the hyperactivity/impulsivity subscale of ADHD RS-IV $(P=0.037)$. After CBT training, the severity of ADHD symptoms assessed by the ADHD RS-IV $(P \leq 0.011)$ and the scores in the subscales of monitor and organization in BRIEF-A $(P<0.006)$ were reduced significantly in adults with ADHD. There were no significant differences in severity of ADHD symptoms or executive functions scored by ADHD RS-IV and BRIEF-A, respectively, between $\mathrm{HC}$ and post-treatment patients (Table 1).

\section{Functional connectivity strength mapping}

Within-group rFCS maps for HC and pre/post-treatment patients

The rFCS patterns were remarkably similar across the HC, pre- and post-treatment patients groups. Regions with high rFCS were predominantly located in default mode network including the medial prefrontal cortex, posterior cingulated cortex/precuneus, and inferior parietal lobule. Moreover, high $\mathrm{rFCS}$ was also observed in lateral prefrontal cortex, lateral temporal cortex, dorsal anterior cingulate cortex, sensorimotor and visual cortices (Fig 1). These results were similar to those found in previous studies (Tomasi \& Volkow, 2010; X. Wang, et al., 2014).

\section{Comparisons between HC and pre-treatment patients}

Compared with $\mathrm{HC}$, the pre-treatment patients presented decreased $\mathrm{rFCS}$ in right superior parietal gyrus (SPG), which overlapped with the region showing CBT-associated increased $\mathrm{rFCS}$ in post-treatment patients than in pre-treatment condition in ADHD (Table 2 and Fig 2B). This result was reproducible after considering the effects of different correlation thresholds (Supporting Information Table I and Table II). No regions showed increased rFCS in pre-treatment patients relative to $\mathrm{HC}$ 
Within-patient treatment effects

Compared with pre-treatment condition, patients showed increased rFCS in bilateral SPG, left middle frontal gyrus, and left posterior lobe of cerebellum (Table 2 and Fig 2A). These results were largely preserved when different correlation thresholds were used (Supporting Information Table I, Table II and Fig I). There were no brain regions showing decreased $\mathrm{rFCS}$ in post-treatment condition in comparison with pre-treatment condition.

\section{Comparisons between $\mathrm{HC}$ and post-treatment patients}

Relative to $\mathrm{HC}$, the post-treatment patients also showed decreased rFCS in right SPG which is very near to that showing difference between $\mathrm{HC}$ and pre-treatment patients and between pre- and post-treatment condition in ADHD (Table 2 and Fig 2C). There were no regions showing increased rFCS in post-treatment patients in comparison with HC. These results were reproducible when the correlation threshold of 0.4 but not 0.2 was used (Supporting Information Table I and Table II). With the latter correlation threshold, we found post-treatment patients showed increased rFCS in left cerebellum posterior lobe compared with HC (Supporting Information Table II).

\section{Seed-based functional connectivity}

We further conducted a seed-based whole-brain functional connectivity analysis by seeding the ROI in areas that simultaneously exhibited between-group differences between $\mathrm{HC}$ vs pre-treatment $\mathrm{ADHD}$ and between post-treatment $\mathrm{ADHD}$ vs pre-treatment ADHD. This procedure identified 60 voxels (centered at the MNI coordinates of $x=15, y=-66, z=66$ ) that was anatomically located in the right SPG (Fig 3A). The results revealed that after the 12-session CBT, the adults with ADHD showed increased FC in left SPG, left superior occipital gyrus, right middle occipital

gyrus and right lingual gyrus with the right SPG (Table 3 and Fig 3B). There were no regions showing decreased FC with the right SPG after CBT in adults with ADHD. 


\section{Correlation analysis}

The CBT-induced increased FC between right SPG and left SPG was positively correlated with the decreased scores of ADHD RS-IV in ADHD $(r=0.79, P=0.007$; Fig 3C). No correlations were found in CBT-induced changes between other clinical variables (i.e., scores of inattention and hyperactivity/impulsivity subscales and BRIEF-A and its factors of organization of material and monitor) and brain measurements (i.e., rFCS and seed-based FC) in areas showing significant condition differences in ADHD.

\section{Discussion}

To our knowledge this is the first study to evaluate the effects of CBT on whole-brain functional connectivity in adults with ADHD. We observed that the CBT not only reduced the severity of ADHD symptoms but also improved the achievement of executive functions, especially in the organization and monitor domains, in adults with ADHD, which were in accordance with the results of previous studies (Stevenson, Whitmont, Bornholt, Livesey, \& Stevenson, 2002; Weiss, et al., 2012). The rFCS analysis indicated treatment-related increased functional connectivity in fronto-parietal network and cerebellum in ADHD. What's more, we specially found that the 12-sessions CBT attenuated the rFCS alterations in the right SPG which showed decreased values in adults with ADHD at baseline. The CBT-associated increased $\mathrm{rFCS}$ in right SPG in ADHD mainly resulted from enhanced functional connectivity within the dorsal attentional network (bilateral SPG and occipital cortex). Importantly, the tighter coupling between bilateral SPG correlated with the improvement of ADHD symptoms in adults with ADHD following CBT.

Before CBT, the adults with ADHD showed lower rFCS in the right SPG compared to $\mathrm{HC}$ at baseline. This result was consistent with a previous study using a similar brain measurement (functional connectivity density) which indicated lower connectivity, especially long-range connectivity, in superior parietal cortex in children with ADHD in a large database (Tomasi \& Volkow, 2012). Task-based fMRI studies also 
identified reduced neural activity in parietal cortex and lower functional connectivity between parietal cortex and other brain regions in ADHD (Cao, et al., 2008; Tamm, Menon, \& Reiss, 2006; Vance, et al., 2007; Vloet, et al., 2010). Considering that the parietal cortex (i.e., the superior parietal cortex) is an important area involved in the dorsal attentional network (Tomasi \& Volkow, 2011), the decreased connectivity between this area with other brain regions found in this study may be associated with the deficits of attention in ADHD. The results from our study and previous studies (Tomasi \& Volkow, 2012; Vance, et al., 2007) indicated that both children and adults with ADHD had lower connectivity in parietal cortex and suggested that the dysfunction in the parietal attentional system may play a significant role in the pathophysiology of ADHD.

In line with previous studies (Stevenson, Whitmont, Bornholt, Livesey, \& Stevenson, 2002; Weiss, et al., 2012), we found that the impact of CBT can be demonstrated in both responses of core symptoms and to cognitive functions in adults with ADHD. On the neural level, adults with ADHD displayed treatment-related increased rFCS in fronto-parietal network (bilateral SPG and left middle frontal gyrus) and cerebellum (left posterior lobe of cerebellum) which showed hypoconnectivity in ADHD in recent r-fMRI studies (Li, et al., 2014; Tomasi \& Volkow, 2012). Previous pharmacological studies revealed that the brain regions which showed increased rFSC in our study were most often affected by medication, including stimulant (i.e., methylphenidate) and atomoxetine, in ADHD (Bush, et al., 2013; Czerniak, et al., 2013; Wong \& Stevens, 2012). A research from Wong and Stevens (2012) found that stimulant medication significantly increased activation and strengthened connectivity in fronto-parietal regions during working memory performance in youths with ADHD. A review aiming to determine the brain regions being modulated by a single dose of methylphenidate identified that the frontal lobe (i.e., the middle and inferior frontal gyri) and cerebellum were most often normalized by increasing activation during task performance due to methylphenidate in ADHD (Czerniak, et al., 2013). A following study showed that atomoxetine was also associated with increased neural activation in 
fronto-parietal networks and cerebellum in ADHD (Bush, et al., 2013). As CBT and medication can both improve core symptoms and cognitive functions in adults with ADHD (Stevenson, Whitmont, Bornholt, Livesey, \& Stevenson, 2002; Weiss, et al., 2012), the similar brain effects of these two treatment approaches may suggest the common neural basis underpinning CBT and medication when administered to adults with ADHD.

The functional connectivity analysis, with the ROI located in the right SPG which showed decreased $\mathrm{rFSC}$ at baseline and increased $\mathrm{rFSC}$ following $\mathrm{CBT}$ in $\mathrm{ADHD}$, found that the coupling within the dorsal attentional network was strengthened following CBT in ADHD. Several structural neuroimaging studies reported the reduction of gray-matter volume and/or cortical thickness in the dorsal attentional network in ADHD (Ahrendts, et al., 2011; Hoekzema, et al., 2012; Proal, et al., 2011). Task-based fMRI revealed deactivation of parietal and occipital regions during performance cognitive tasks in ADHD (Silk, et al., 2005; Vance, et al., 2007). In addition, reduced functional connectivity of the dorsal attentional network has been found in r-fMRI study in adults with childhood ADHD (McCarthy, et al., 2013). Recently, researches using graph analysis indicated reduced nodal efficiency in occipital cortex (L. Wang, et al., 2009), reduced functional connectivity density in parietal lobe (Tomasi \& Volkow, 2012) and lower pair-wise connectivity in an inner-network, encompassing right parietal and temporal lobes and left occipital lobe (Xia, Foxe, Sroubek, Branch, \& Li, 2014) in ADHD. Considering the important role of the dorsal attentional network in maintaining attention and suppressing attention to irrelevant stimuli (Capotosto, Babiloni, Romani, \& Corbetta, 2009; Shulman, et al., 2009), hypoconnectivity within this network in ADHD would contribute to the core deficits of ADHD and strengthening the coupling within this network would alleviate the symptoms of ADHD. The correlation analysis in our study, which found that the increased value of the connectivity between bilateral SPG have a positive correlation with the decreased symptoms of ADHD measured by ADHD RS-IV in ADHD, supported this inference. 
There are several issues need to be further addressed. First, as it is an open-label and nonrandomized study, we cannot clearly determine whether the rFCS change over time in the adults with ADHD were due to CBT-specific or placebo effects. A randomized, controlled study including an active control condition (i.e., non-CBT treatment condition) or wait-list patients with brain imaging data acquired with the same time interval as control group would be helpful to clarify this issue. Second, the healthy participants serving as controls in this study scanned only once, so we cannot exclude the possibility of potential contribution of natural neural development course to the observed effects following CBT in ADHD. The time comparable scans in healthy controls were needed to clarify this issue. Third, the sample size of this study was small and thus reduced the statistical power in revealing subtle effects. Further large-sample studies are warranted to test the reproducibility of the current findings. Forth, the diagnosis of adult with ADHD in this study was based on the patients' self-reported symptoms in childhood and adult, and all patients fulfilled diagnostic criteria for adult ADHD according to CAARID. Nevertheless, potential recall bias associated with retrospective diagnoses of ADHD in childhood may not be completely avoided in these patients. Including patients with childhood ADHD can help to address this issue.

In conclusion, our study demonstrated that, after a 12-session CBT, adults with ADHD showed increased whole-brain functional connectivity in the fronto-parietal network and cerebellum. These brain regions were also mostly affected by medication in ADHD. What's more, following CBT, ADHD strengthen functional coupling within dorsal attentional network (i.e., bilateral SPG and occipital cortex), and the increased functional connectivity between bilateral SPG have a positive correlation with the improvement of ADHD symptoms. Our findings provide evidence that CBT can selectively modulate the intrinsic network connectivity in the fronto-parietal network and cerebellum and suggest that the CBT may share common brain mechanism with the pharmacology in adults with ADHD. 


\section{Acknowledgements}

This work was supported by the National Key Basic Research Program of China (973

Program, 2014CB846104) and the National Science Foundation of China (Nos. $81471382,81020108022,81000593$ and 81371496).

\section{Appendix A. Supplementary data}




\section{References}

Ahrendts, J., Rusch, N., Wilke, M., Philipsen, A., Eickhoff, S. B., Glauche, V., Perlov, E., Ebert, D., Hennig, J., \& van Elst, L. T. (2011). Visual cortex abnormalities in adults with ADHD: a structural MRI study. World J Biol Psychiatry, 12, 260-270.

Biederman, J., Petty, C. R., Monuteaux, M. C., Fried, R., Byrne, D., Mirto, T., Spencer, T., Wilens, T. E., \& Faraone, S. V. (2010). Adult Psychiatric Outcomes of Girls With Attention Deficit Hyperactivity Disorder: 11-Year Follow-Up in a Longitudinal Case-Control Study. Am J Psychiatry.

Brown, T. E. (2008). ADD/ADHD and Impaired Executive Function in Clinical Practice. Curr Psychiatry Rep, 10, 407-411.

Bush, G., Holmes, J., Shin, L. M., Surman, C., Makris, N., Mick, E., Seidman, L. J., \& Biederman, J. (2013). Atomoxetine increases fronto-parietal functional MRI activation in attention-deficit/hyperactivity disorder: a pilot study. Psychiatry Res, 211, 88-91.

Cao, Q., Zang, Y., Zhu, C., Cao, X., Sun, L., Zhou, X., \& Wang, Y. (2008). Alerting deficits in children with attention deficit/hyperactivity disorder: Event-related $\mathrm{AMRI}$ evidence. Brain Res.

Capotosto, P., Babiloni, C., Romani, G. L., \& Corbetta, M. (2009). Frontoparietal cortex controls spatial attention through modulation of anticipatory alpha rhythms. J Neurosci, 29, 5863-5872.

Castellanos, F. X., \& Proal, E. (2012). Large-scale brain systems in ADHD: beyond the prefrontal-striatal model. Trends Cogn Sci, 16, 17-26.

Chao-Gan, Y., \& Yu-Feng, Z. (2010). DPARSF: A MATLAB Toolbox for "Pipeline" Data Analysis of Resting-State fMRI. Front Syst Neurosci, 4, 13.

Choi, J., Jeong, B., Lee, S. W., \& Go, H. J. (2013). Aberrant development of functional connectivity among resting state-related functional networks in medication-naive ADHD children. PLoS ONE, 8, e83516.

Cocchi, L., Bramati, I. E., Zalesky, A., Furukawa, E., Fontenelle, L. F., Moll, J., Tripp, G., \& Mattos, P. (2012). Altered functional brain connectivity in a non-clinical sample of young adults with attention-deficit/hyperactivity disorder. J Neurosci, 32, 17753-17761.

Conners, C. K., Erhardt, D., \& Sparrow, E. (1999). Conner's Adult ADHD Rating Scales: CAARS. In: MHS Toronto.

Czerniak, S. M., Sikoglu, E. M., King, J. A., Kennedy, D. N., Mick, E., Frazier, J., \& Moore, C. M. (2013). Areas of the brain modulated by single-dose methylphenidate treatment in youth with ADHD during task-based fMRI: a systematic review. Harv Rev Psychiatry, 21, 151-162.

Diagnostic and statistical manual of mental disorders, text revision (DSM-IV-TR). (2000). American Psychiatric Association.

DuPaul, G., Power, T. J., Anastopoulos, A. D., \& Reid, R. (1998). ADHD Rating Scale-IV: Checklists, Norms, and Clinical Interpretations. In: New York, Guilford.

Emilsson, B., Gudjonsson, G., Sigurdsson, J. F., Baldursson, G., Einarsson, E., Olafsdottir, H., \& Young, S. (2011). Cognitive behaviour therapy in medication-treated adults with ADHD and persistent symptoms: a randomized controlled trial. BMC Psychiatry, 11, 116.

First, M. B., Spitzer, R. L., Gibbon, M., \& Williams, J. B. (2012). Structured Clinical Interview for DSM-IV® Axis I Disorders (SCID-I), Clinician Version, Administration Booklet: American Psychiatric Pub.

Gioia, G. A., Isquith, P. K., Guy, S. C., \& Gerard, K. (2000). Behavior rating inventory of executive function. Neuropsychol Dev Cogn., 6, 235-238. 
Hoekzema, E., Carmona, S., Ramos-Quiroga, J. A., Richarte Fernandez, V., Picado, M., Bosch, R., Soliva, J. C., Rovira, M., Vives, Y., Bulbena, A., Tobena, A., Casas, M., \& Vilarroya, O. (2012). Laminar thickness alterations in the fronto-parietal cortical mantle of patients with attention-deficit/hyperactivity disorder. PLoS ONE, 7, e48286.

Krain, A. L., \& Castellanos, F. X. (2006). Brain development and ADHD. Clin Psychol Rev, 26, 433-444.

Li, F., He, N., Li, Y., Chen, L., Huang, X., Lui, S., Guo, L., Kemp, G. J., \& Gong, Q. (2014). Intrinsic brain abnormalities in attention deficit hyperactivity disorder: a resting-state functional MR imaging study. Radiology, 272, 514-523.

Lofthouse, N., Arnold, L. E., Hersch, S., Hurt, E., \& DeBeus, R. (2012). A review of neurofeedback treatment for pediatric ADHD. J Atten Disord, 16, 351-372.

McCarthy, H., Skokauskas, N., Mulligan, A., Donohoe, G., Mullins, D., Kelly, J., Johnson, K., Fagan, A., Gill, M., Meaney, J., \& Frodl, T. (2013). Attention network hypoconnectivity with default and affective network hyperconnectivity in adults diagnosed with attention-deficit/hyperactivity disorder in childhood. JAMA Psychiatry, 70, 1329-1337.

Miller, T. W., Nigg, J. T., \& Faraone, S. V. (2007). Axis I and II comorbidity in adults with ADHD. J Abnorm Psychol, 116, 519-528.

Murphy, K., Birn, R. M., Handwerker, D. A., Jones, T. B., \& Bandettini, P. A. (2009). The impact of global signal regression on resting state correlations: are anti-correlated networks introduced? Neuroimage, 44, 893-905.

Proal, E., Reiss, P. T., Klein, R. G., Mannuzza, S., Gotimer, K., Ramos-Olazagasti, M. A., Lerch, J. P., He, Y., Zijdenbos, A., Kelly, C., Milham, M. P., \& Castellanos, F. X. (2011). Brain gray matter deficits at 33-year follow-up in adults with attention-deficit/hyperactivity disorder established in childhood. Arch Gen Psychiatry, 68, 1122-1134.

Ramsay, J. R. (2007). Current status of cognitive-behavioral therapy as a psychosocial treatment for adult attention-deficit/hyperactivity disorder. Curr Psychiatry Rep, 9, 427-433.

Safren, S. A. (2006). Cognitive-behavioral approaches to ADHD treatment in adulthood. J Clin Psychiatry, 67 Suppl 8, 46-50.

Safren, S. A., Otto, M. W., Sprich, S., Winett, C. L., Wilens, T. E., \& Biederman, J. (2005). Cognitive-behavioral therapy for ADHD in medication-treated adults with continued symptoms. Behav Res Ther, 43, 831-842.

Safren, S. A., Perlman, C. A., Sprich, S., \& Otto, M. W. (2005). Mastering your adult ADHD: A cognitive-behavioral treatment program therapist guide: Oxford university press.

Safren, S. A., Sprich, S., Mimiaga, M. J., Surman, C., Knouse, L., Groves, M., \& Otto, M. W. (2010). Cognitive behavioral therapy vs relaxation with educational support for medication-treated adults with ADHD and persistent symptoms: a randomized controlled trial. JAMA, 304, $875-880$.

Shulman, G. L., Astafiev, S. V., Franke, D., Pope, D. L., Snyder, A. Z., McAvoy, M. P., \& Corbetta, M. (2009). Interaction of stimulus-driven reorienting and expectation in ventral and dorsal frontoparietal and basal ganglia-cortical networks. J Neurosci, 29, 4392-4407.

Silk, T., Vance, A., Rinehart, N., Egan, G., O'Boyle, M., Bradshaw, J. L., \& Cunnington, R. (2005). Fronto-parietal activation in attention-deficit hyperactivity disorder, combined type: functional magnetic resonance imaging study. Br J Psychiatry, 187, 282-283.

Solanto, M. V., Marks, D. J., Wasserstein, J., Mitchell, K., Abikoff, H., Alvir, J. M., \& Kofman, M. D. 
(2010). Efficacy of meta-cognitive therapy for adult ADHD. Am J Psychiatry, 167, 958-968.

Song, X. W., Dong, Z. Y., Long, X. Y., Li, S. F., Zuo, X. N., Zhu, C. Z., He, Y., Yan, C. G., \& Zang, Y. F. (2011). REST: a toolkit for resting-state functional magnetic resonance imaging data processing. PLoS ONE, 6, e25031.

Stevenson, C. S., Whitmont, S., Bornholt, L., Livesey, D., \& Stevenson, R. J. (2002). A cognitive remediation programme for adults with Attention Deficit Hyperactivity Disorder. Aust N Z J Psychiatry, 36, 610-616.

Stavro, G. M., Ettenhofer, M. L., \& Nigg, J. T. (2007). Executive functions and adaptive functioning in young adult attention-deficit/hyperactivity disorder. J Int Neuropsychol Soc, 13, 324-334.

Tamm, L., Menon, V., \& Reiss, A. L. (2006). Parietal attentional system aberrations during target detection in adolescents with attention deficit hyperactivity disorder: event-related fMRI evidence. Am J Psychiatry, 163, 1033-1043.

Tomasi, D., \& Volkow, N. D. (2010). Functional connectivity density mapping. Proc Natl Acad Sci U S A, 107, 9885-9890.

Tomasi, D., \& Volkow, N. D. (2011). Association between functional connectivity hubs and brain networks. Cereb Cortex, 21, 2003-2013.

Tomasi, D., \& Volkow, N. D. (2012). Abnormal functional connectivity in children with attention-deficit/hyperactivity disorder. Biol Psychiatry, 71, 443-450.

Vance, A., Silk, T. J., Casey, M., Rinehart, N. J., Bradshaw, J. L., Bellgrove, M. A., \& Cunnington, R. (2007). Right parietal dysfunction in children with attention deficit hyperactivity disorder, combined type: a functional MRI study. Mol Psychiatry, 12, 826-832, 793.

Vloet, T. D., Gilsbach, S., Neufang, S., Fink, G. R., Herpertz-Dahlmann, B., \& Konrad, K. (2010). Neural mechanisms of interference control and time discrimination in attention-deficit/hyperactivity disorder. J Am Acad Child Adolesc Psychiatry, 49, 356-367.

Wang, L., Zhu, C., He, Y., Zang, Y., Cao, Q., Zhang, H., Zhong, Q., \& Wang, Y. (2009). Altered small-world brain functional networks in children with attention-deficit/hyperactivity disorder. Hum Brain Mapp, 30, 638-649.

Wang, X., Xia, M., Lai, Y., Dai, Z., Cao, Q., Cheng, Z., Han, X., Yang, L., Yuan, Y., Zhang, Y., Li, K., Ma, H., Shi, C., Hong, N., Szeszko, P., Yu, X., \& He, Y. (2014). Disrupted resting-state functional connectivity in minimally treated chronic schizophrenia. Schizophr Res, 156, $150-156$.

Weiss, M., Murray, C., Wasdell, M., Greenfield, B., Giles, L., \& Hechtman, L. (2012). A randomized controlled trial of CBT therapy for adults with ADHD with and without medication. BMC Psychiatry, 12, 30.

Wong, C. G., \& Stevens, M. C. (2012). The effects of stimulant medication on working memory functional connectivity in attention-deficit/hyperactivity disorder. Biol Psychiatry, 71, 458-466.

Xia, S., Foxe, J. J., Sroubek, A. E., Branch, C., \& Li, X. (2014). Topological organization of the "small-world" visual attention network in children with attention deficit/hyperactivity disorder (ADHD). Front Hum Neurosci, 8, 162.

Young, S., Khondoker, M., Emilsson, B., Sigurdsson, J. F., Philipp-Wiegmann, F., Baldursson, G., Olafsdottir, H., \& Gudjonsson, G. (2015). Cognitive-behavioural therapy in medication-treated adults with attention-deficit/hyperactivity disorder and co-morbid psychopathology: a randomized controlled trial using multi-level analysis. Psychol Med, 45, 2793-2804. 
Table 1. Demographic, clinical and neuropsychological characteristics for adults with ADHD and healthy controls

\begin{tabular}{lcccccc}
\hline & $\mathrm{HC}$ & $\begin{array}{c}P \\
\text { pre-CBT })\end{array}$ & $\begin{array}{c}\text { ADHD } \\
\text { (pre-CBT) }\end{array}$ & $\begin{array}{c}P(\mathrm{HC} \text { vs } \\
\text { post-CBT) }\end{array}$ & $\begin{array}{c}\text { ADHD } \\
\text { post-CBT }\end{array}$ & $\begin{array}{c}P \text { (pre- vs } \\
\text { post-CBT) }\end{array}$ \\
\hline age(years) & $24.8 \pm 4.0$ & 0.49 & $26.0 \pm 4.5$ & & & \\
gender(female/male) & $4 / 8$ & 0.83 & $5 / 5$ & & & \\
IQ & $124.7 \pm 7.2$ & 0.63 & $122.6 \pm 12.4$ & & & \\
ADHD RS-IV & $28.0 \pm 5.8$ & $<0.001$ & $43.0 \pm 7.1$ & 0.361 & $30.5 \pm 6.8$ & $<0.001$ \\
$\quad$ Inattention & $15.1 \pm 3.0$ & $<0.001$ & $25.8 \pm 3.8$ & 0.053 & $18.2 \pm 3.9$ & 0.004 \\
$\quad$ Hyperactivity/impulsivity & $12.9 \pm 3.4$ & 0.037 & $17.2 \pm 5.5$ & 0.71 & $12.3 \pm 4.2$ & 0.011 \\
BRIEF-A & $91.7 \pm 19.2$ & $<0.001$ & $133.6 \pm 23.5$ & 0.067 & $107.2 \pm 18.2$ & 0.011 \\
Inhibit & $11.3 \pm 2.6$ & $<0.001$ & $15.7 \pm 3.8$ & 0.357 & $12.5 \pm 3.4$ & 0.009 \\
Shift & $9.0 \pm 2.7$ & 0.005 & $11.8 \pm 3.0$ & 0.062 & $10.1 \pm 2.1$ & 0.082 \\
Emotional Control & $14.9 \pm 3.8$ & 0.001 & $18.3 \pm 5.3$ & 0.638 & $16.3 \pm 5.1$ & 0.362 \\
Monitor & $9.3 \pm 1.4$ & $<0.001$ & $14.8 \pm 2.4$ & 0.099 & $11.3 \pm 2.2$ & 0.005 \\
Initiate & $12.0 \pm 2.8$ & $<0.001$ & $18.8 \pm 2.7$ & 0.015 & $15.0 \pm 2.5$ & 0.01 \\
$\quad$ Working Memory & $10.9 \pm 3.2$ & $<0.001$ & $18.4 \pm 4.0$ & 0.03 & $15.3 \pm 2.8$ & 0.076 \\
$\quad$ Plan & $14.1 \pm 3.1$ & $<0.001$ & $21.7 \pm 5.4$ & 0.232 & $16.5 \pm 3.8$ & 0.017 \\
$\quad$ Organization & $11.4 \pm 2.9$ & 0.001 & $18.5 \pm 4.5$ & 0.142 & $13.7 \pm 3.8$ & $<0.001$ \\
\hline
\end{tabular}

Abbreviations: ADHD, attention-deficit/hyperactivity disorder; CBT, cognitive-behavioral therapy; HC, healthy control; ADHD RS-IV, ADHD Rating Scale-IV; BRIEF-A, behavior rating inventory of executive function, adult version 
Table 2. CBT-related regional functional connectivity strength changes within and between groups.

\begin{tabular}{lccccccc}
\hline \multicolumn{1}{c}{ Areas } & L/R & Cluster size & Brodmann & \multicolumn{4}{c}{ Peak MNI coordinates } \\
\multicolumn{1}{c}{ (voxels) } & area & x & y & z & t-value \\
\hline Post-CBT $>$ Pre-CBT & & & & & & & \\
superior parietal gyrus & $\mathrm{R}$ & 189 & 7 & 27 & -60 & 66 & 4.61 \\
superior parietal gyrus & $\mathrm{L}$ & 284 & 7 & -24 & -66 & 45 & 5.76 \\
$\begin{array}{l}\text { Middle frontal gurus } \\
\text { cerebellum posterior lobule }\end{array}$ & $\mathrm{L}$ & 190 & 9 & -45 & 27 & 24 & 5.63 \\
HC $>$ Pre-CBT & $\mathrm{L}$ & 291 & & -42 & -69 & -51 & 7.09 \\
$\begin{array}{l}\text { Superior parietal gyrus } \\
\text { HC }>\text { Post-CBT }\end{array}$ & $\mathrm{R}$ & 125 & 7 & 15 & -66 & 54 & 5.72 \\
Superior parietal gyrus & $\mathrm{R}$ & 132 & 7 & 6 & -75 & 54 & 4.04 \\
\hline
\end{tabular}

Abbreviations: CBT, cognitive-behavioral therapy; HC, healthy control; L, left; R, right; MNI, Montreal Neurological Institute 
Table 3. Regions showing increased functional connectivity of the right superior parietal gyrus-ROIs between pre- and post-CBT in adults with ADHD.

\begin{tabular}{lccccccc}
\hline \multicolumn{1}{c}{ Areas } & Cluster size & Brodmann & \multicolumn{4}{c}{ Peak MNI coordinates } \\
& L/R & (voxels) & area & x & y & z & t-value \\
\hline Lingual gyrus & $\mathrm{R}$ & 160 & $17 / 18$ & 0 & -81 & -3 & 4.88 \\
Superior occipital gyrus & $\mathrm{L}$ & 232 & $18 / 19$ & -24 & -84 & 24 & 4.87 \\
Middle Occipital gyrus & $\mathrm{R}$ & 219 & $18 / 19$ & 30 & -87 & 9 & 5.09 \\
Superior parietal gyrus & $\mathrm{L}$ & 251 & $5 / 7 / 40$ & -24 & -57 & 57 & 6.55 \\
\hline
\end{tabular}

Abbreviations: ROI, region of interest; L, left; R, right; MNI, Montreal Neurological Institute 


\section{Figure legends}

Figure 1. Within-group regional functional connectivity strength maps for healthy control and pre- and post-treatment ADHD. HC, healthy control; CBT, cognitive-behavioral therapy; L, left; R, right.

Figure 2. Significant pre-/post-treatment conditions and between-group differences in regional functional connectivity strength. $\mathrm{HC}$, healthy control; $\mathrm{CBT}$, cognitive-behavioral therapy.

Figure 3. Cognitive-behavioral therapy (CBT)-associated increased functional connectivity with the right superior parietal gyrus in adults with ADHD. (A) The axial image shows the anatomical location of the right superior parietal gyrus seed. (B) Brain regions showing increased functional connectivity with right superior parietal gyrus following $\mathrm{CBT}$ in adults with ADHD. a, right lingual gyrus; b, right middle occipital gyrus; c, left superior occipital gyrus; d, left superior parietal gyrus. The numbers at the lower of axial images refer to the MNI z-coordinates. (C) The scatter map shows significant correlation between changes of functional connectivity between bilateral superior parietal gyrus and scores of ADHD RS-IV following CBT in adults with ADHD. 


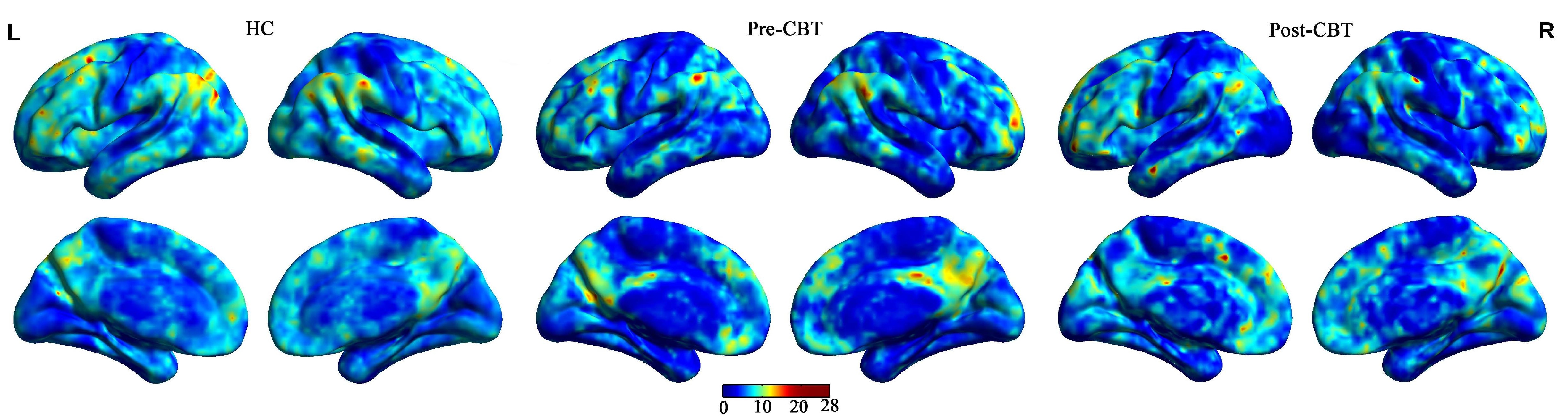



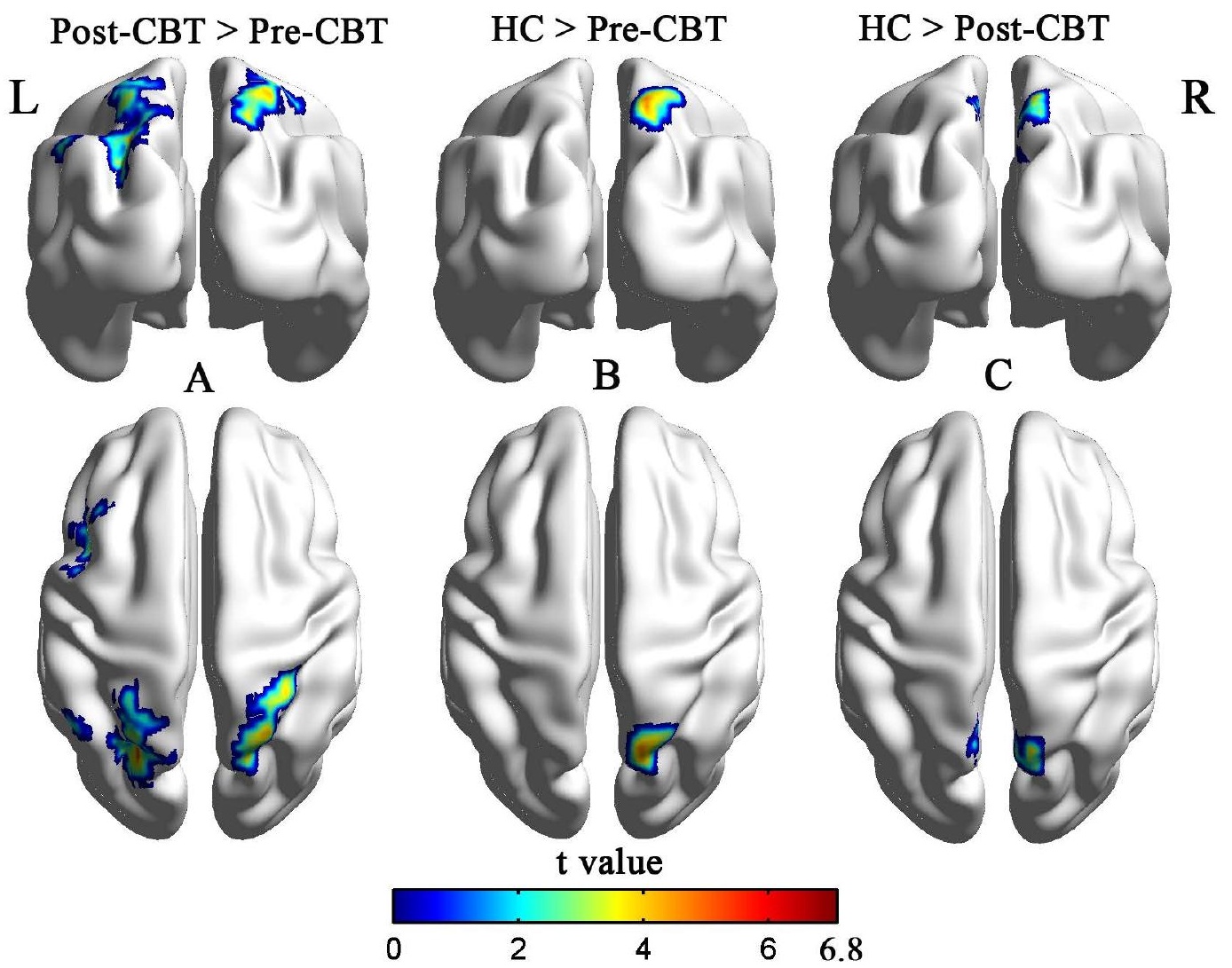
A
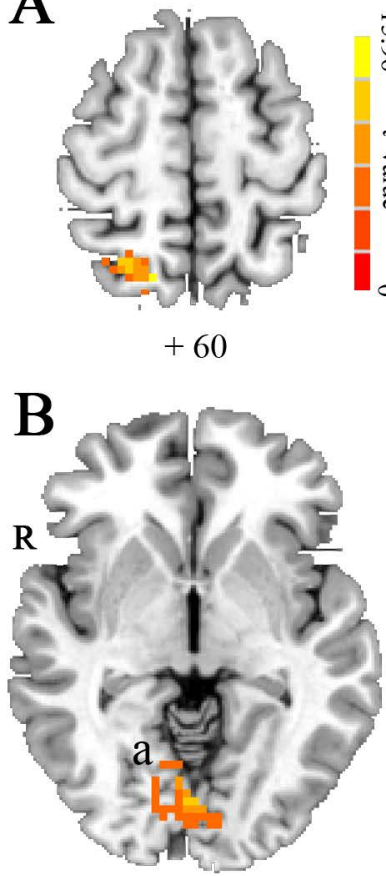

$-3$
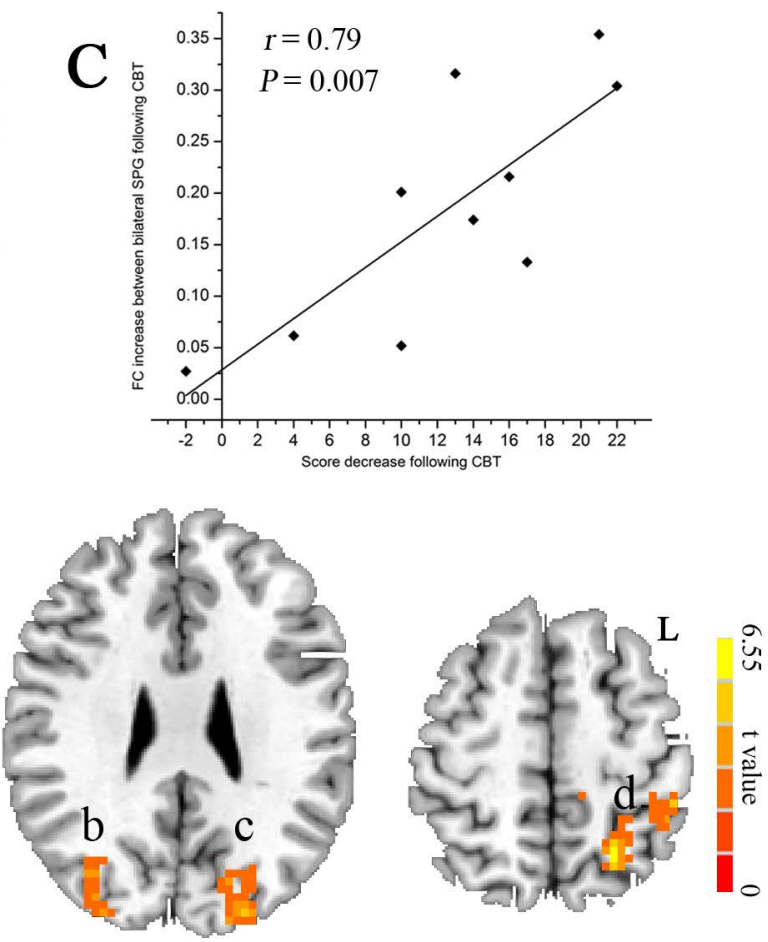

$+27$

$+58$ 